\title{
PIODERMA GANGRENOSO EM ASSOCIAÇÃO A HIDROSADENITE SUPURATIVA - UMA ASSOCIAÇÃO RARA MAS BEM DEFINIDA
}

\author{
António Fernandes Massa 1,*,\#, João Borges da Costa 2,\#, Paulo Filipe 3,\#, Manuel Sacramento Marques",\# \\ 'Interno do Internato de Dermatovenereologia/Resident, Dermatology and Venereology \\ ${ }^{2}$ Assistente HospitalarGraduado de Dermatovenerologia, Serviço de Dermatologia, CHLN/Graduated Consultant \\ of Dermatology, Dermatology Department, CHLN, Lisboa; Professor Auxiliar Convidado de Dermatologia da FML/ \\ Professor of Dermatology and Venereology \\ ${ }^{3}$ Professor Doutor do Serviço de Dermatologia e Venereologia/Professor of Dermatology and Venereology \\ ${ }^{4}$ Director de Serviço, Serviço de Dermatologia/Chief of Dermatology and Head of the Dermatology Department \\ * Serviço de Dermatologia, Centro Hospitalar V.N.Gaia/Espinho, EPE, Vila Nova de Gaia, Portugal \\ "Serviço de Dermatologia, Hospital de Santa Maria - Centro Hospitalar de Lisboa Norte, EPE, Lisboa, Portugal
}

RESUMO - O pioderma gangrenoso (PG) e a hidrosadenite supurativa (HS) são doenças inflamatórias, de etiologia desconhecida, sendo a sua associação rara mas bem documentada. Descreve-se o caso de uma doente de 27 anos, com história de lesões axilares e inguinais de HS desde há 4 anos com desenvolvimento de uma lesão de PG na perna direita. Realizou-se tratamento com corticoterapia sistémica, em internamento. Posteriormente realizou tratamento com clindamicina e rifampicina, seguida de isotretínoina oral em baixa dose, com boa resposta.

A associação da HS e do PG poderá ter uma origem comum relacionada com alterações da atividade dos neutrófilos. A descrição do síndrome autossómico dominante PAPA (PG, acne e artrite piogénica) assim como PASH (PG, acne e HS), dermatoses enquadradas no espectro das doenças autoinflamatórias, perspectivam uma maior compreensão da etiopatogenia desta associação com possíveis implicações diagnósticas e terapêuticas.

PALAVRAS-CHAVE - Pioderma gangrenoso; Hidrosadenite supurativa.

\section{PYODERMA GANGRENOSUM ASSOCIATED WITH HIDRADENITIS SUPURATIVA - A RARE BUT WELL ESTABLISHED ASSOCIATION}

\begin{abstract}
Pyoderma gangrenosum (PG) and hidradenitis suppurativa (HS) are inflammatory diseases of unknown aetiology, which association is rare but well established. We describe the case of a 27-year-old female patient, with axillary and inguinal hidradenitis supurativa lesions since four years ago who developed a pyoderma gangrenosum lesion in her right leg. Treatment was instituted with systemic corticosteroids in inpatient setting. Afterwards, clindamycin and rifampicin were chosen followed by low dose isotretinoin with good response.

The association of HS and PG can possibly have a common aetiology related to neutrophilic activity dysfunction. The description autossomic dominant syndrome PAPA (PG, acne and pyogenic arthritis) as well as PASH (PG, acne and HS), dermatos included in the spectrum of autoinflammatory diseases, promise a better comprehension of aetiopathogenesis of this association with possible diagnostic and therapeutic improvements.
\end{abstract}

KEY-WORDS - Pyoderma gangrenosum; Hidradenitis suppurativa. 


\title{
Caso Clínico
}

\author{
Conflitos de interesse: Os autores declaram não possuir conflitos de interesse. \\ No conflicts of interest. \\ Suporte financeiro: $O$ presente trabalho não foi suportado por nenhum subsídio ou bolsa. \\ No sponsorship or scholarship granted. \\ Direito à privacidade e consentimento escrito / Privacy policy and informed consent: Os autores declaram \\ que pediram consentimento ao doente para usar as imagens no artigo. The authors declare that the patient gave \\ written informed consent for the use of its photos in this article.
}

Recebido/Received - Abril/April 2014; Aceite/Accepted - Maio/May 2014

Correspondêncią:

Dr. António F. Massa

Serviço de Dermatologia

Centro Hospitalar V.N.Gaia/Espinho, EPE

R. Conceição Fernandes

4430 Vila Nova de Gaia, Portugal

Email: antoniofmassa@gmail.com

\section{INTRODUÇÃO}

O pioderma gangrenoso (PG) e a hidrosadenite supurativa (HS) são doenças inflamatórias raras, cuja associação está descrita em cerca de 30 casos'.

O Pioderma gangrenoso é uma dermatose neutrofílica, de etiologia desconhecida, caracterizando-se pelo desenvolvimento de um nódulo ou pústula dolorosa formando progressivamente uma úlcera com bordo elevado e de aspecto denteado. Surge mais frequentemente nas extremidades inferiores mas pode surgir em qualquer local do corpo'. Foi descrito pela primeira vez em 1916 por Brocq como phagédenisme géometri$q^{2} e^{2}$ e em 1930 por Burnsting e colaboradores como pioderma gangrenoso ${ }^{3}$, sendo realçado o componente de úlceras necrotizantes. A associação com outras doenças sistémicas surge em metade dos casos, como a doença inflamatória intestinal, a gamapatia monoclonal e o mieloma ${ }^{4}$. Na patogénese estão implicadas alterações da quimiotaxia, e da reactividade neutrofíli$\mathrm{ca}^{5}$ bem como de linfócitos T auxiliares e citotóxicos ${ }^{6}$.

A HS é uma dermatose neutrofílica crónica e recorrente, de etiologia desconhecida. Surge após a puberdade, com lesões dolorosas, inflamatórias, nodulares, fistulizantes e cicatriciais nas áreas com glândulas apócrinas $^{7}$. Observaram-se neutrófilos disfuncionais ${ }^{8}$ assim como a possível influência hormonal e genética 9 .

A única ligação comum sugerida entre estas duas entidades parece ser uma função neutrofílica defeituosa embora não exista evidência de suporte de que este seja o factor etiológico comum ${ }^{10}$.

\section{CASO CLÍNICO}

Doente do sexo feminino, caucasiana, de 27 anos de idade que recorreu ao serviço de urgência devido a ulceração na perna direita, com duas semanas de evolução, tendo tido início como nódulo eritematoso doloroso (Fig. 1). Realizada limpeza cirúrgica com agravamento da lesão (Fig. 2). Instituída terapêutica antibiótica com flucloxacilina e meropenem. Transferida para o serviço de Dermatologia, apresentando à entrada lesão ulcerada na perna direita com $10 \mathrm{~cm}$ por $6 \mathrm{~cm}$, com bordos relativamente bem definidos, irregulares, violáceos com aspecto em mordida e exsudação ligeira, tendo-se diagnosticado pioderma gangrenoso. Obesa.

Hidrosadenite supurativa desde há 4 anos, com lesões axilares e inguinais - estádio III de Hurley (Fig. 4). Há 2 anos tinha realizado tratamento com isotretinoína

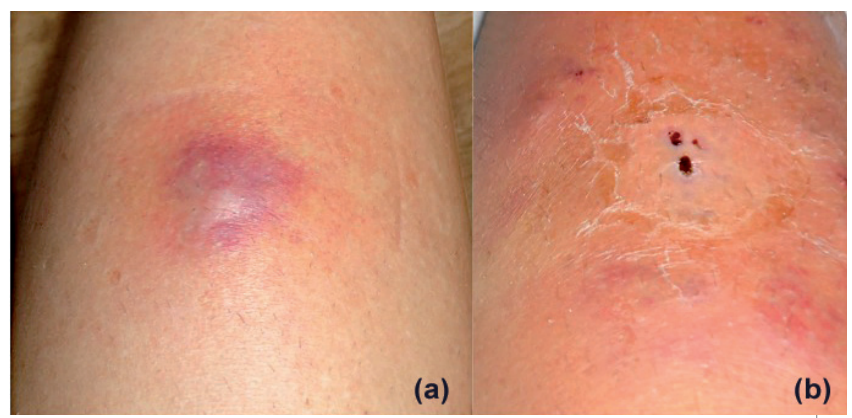

Fig 1 - (a) Nódulo doloroso de coloração violácea (b) com início de ulceração. 


\section{Caso Clínico}

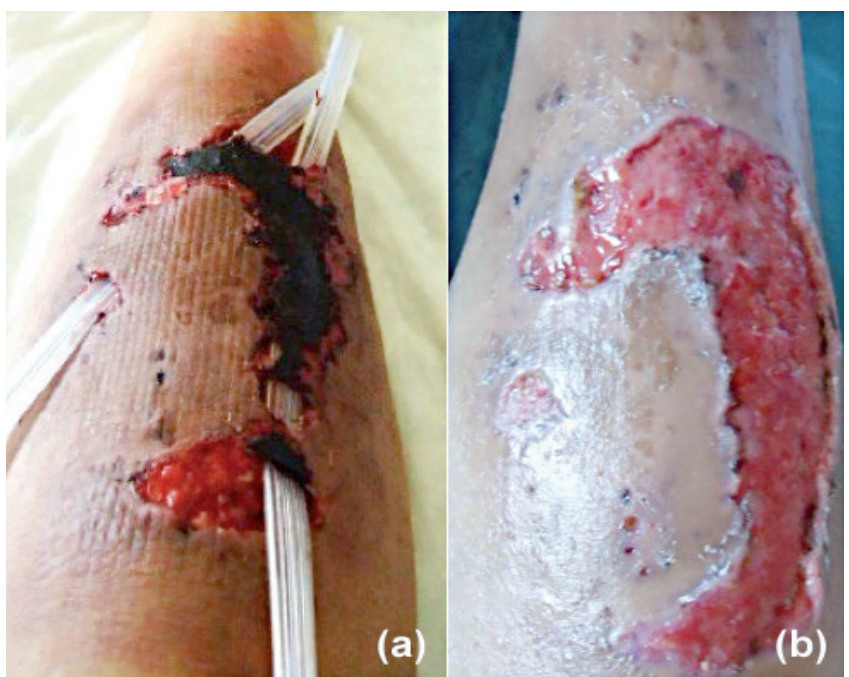

Fig 2 - (a) Lesão ulcerada após limpeza cirúrgica e (b) à entrada do serviço de Dermatologia.

oral $20 \mathrm{mg} /$ dia e clindamicina tópica durante 1 ano, sem melhoria.

Sem história de traumatismo. Negou alterações do trânsito intestinal, oculares e articulares. Fenómeno de patergia positivo.

Analiticamente, sem anemia ou leucocitose. Ausência de pico monoclonal. $O$ esfregaço sanguíneo não apresentava alterações. Rastreio de autoimunidade negativo.

Realizou colonoscopia total que não demonstrou alterações do tracto gastrointestinal. Telerradiografia de tórax normal. Hemoculturas negativas. Serologias para $\circ \mathrm{VIH}$, anti- VHC, AgHBs, AcHBs negativas. RPR

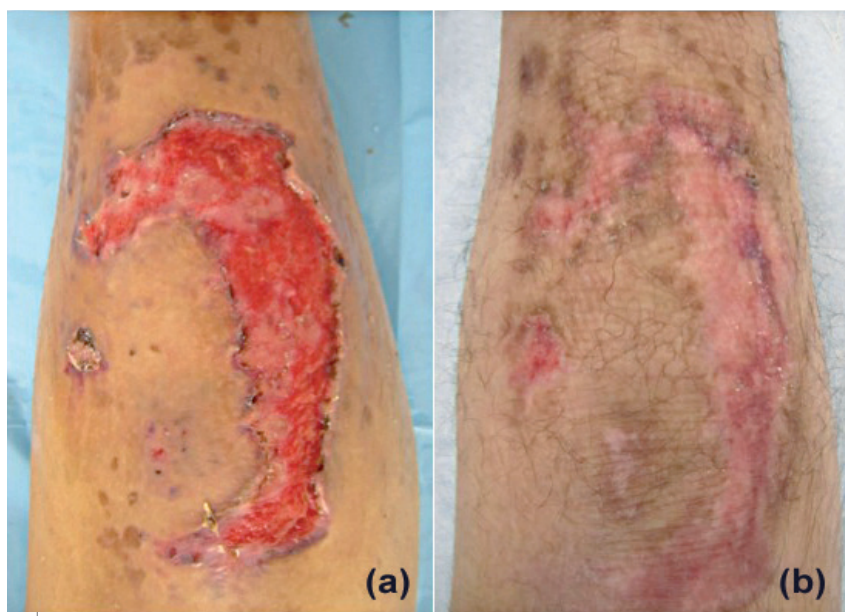

Fig 3 - (a) Lesão em reepitelização (b) lesão reepitelizada.

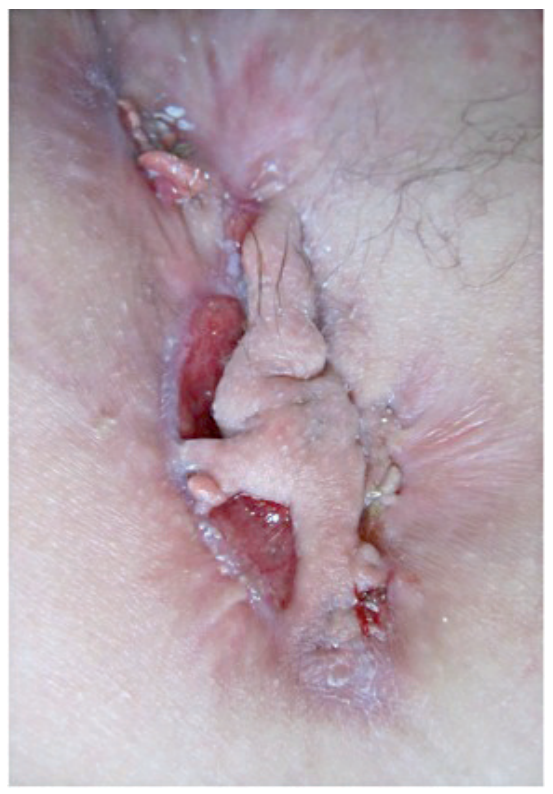

Fig 4 - Lesão axilar com trajectos fistulosos vários.

negativo. Culturas micológicas e bacteriológicas da lesão foram negativas.

Sem história de acne grave. Sem história familiar de autoimunidade ou de doença inflamatória intestinal.

Realizou-se tratamento com prednisolona $1 \mathrm{mg} / \mathrm{Kg} /$ dia com boa evolução e reepitelização da lesão de pioderma gangrenoso.

Seguidamente, realizou o esquema de Mendonça e Griffiths ${ }^{11}$, clindamicina $300 \mathrm{mg} 2 x /$ dia e rifampicina $300 \mathrm{mg} 2 x /$ dia, durante 10 semanas, com estabilização das lesões axilares e inguinais. Instituiu-se terapêutica com isotretinoína oral em baixa dose ( $10 \mathrm{mg} / \mathrm{dia})$. Realizado tratamento cirúrgico com excisão alargada das lesões inguinais.

Em seguimento desde há 2 anos, sem recidiva de lesões de pioderma gangrenoso e com um ano de seguimento após o tratamento cirúrgico das lesões inguinais, mantendo-se desde então sem actividade da HS, não realizando actualmente qualquer medicação para o efeito.

\section{DISCUSSÃO}

O diagnóstico prévio de HS ao desenvolvimento de PG na nossa doente corrobora o descrito na literatura. A HS antecede por período varíavel o PG, estando descritos longos períodos de latência, com uma média de 21 anos por Ah-Weng e colaboradores ${ }^{10}$ e períodos 


\section{Caso Clínico}

mais curtos, com um tempo de latência média de 3 anos por Hsiao e colaboradores'. Nesta série, a maior descrita, com onze casos desta associação, a localização das lesões de PG observadas foi mais comum nos mesmos locais das lesões de HS, o que pode conferir alguma indefinição diagnóstica, visto que lesões de HS com ulceração crónica podem assemelhar-se a lesões de PG e a distinção histológica entre estas duas entidades pode ser difícil. Foi descrita uma baixa prevalência de doença inflamatória intestinal nesta série, e uma predominância no sexo feminino, que poderá estar relacionada com a maior prevalência de HS no sexo feminino $^{12}$. A maior parte dos doentes observados eram obesos ${ }^{1}$, tal como neste caso, o que poderá estar relacionado com o potencial de exacerbação da HS devido ao aumento de forças de cisalhamento, oclusão e maceração ${ }^{13,14}$ e pela criação de um estado de hiperandrogenismo ${ }^{9}$, sendo que uma diminuição de peso pode levar a melhoria da $\mathrm{HS}^{15}$. Foi reportado aumento dos marcadores de inflamação, e também comorbilidades tais como obesidade, diabetes mellitus tipo II e doença arterial coronária'.

No entanto, não se observa correlação da actividade da doença nas duas entidades antes ou depois do desenvolvimento do pioderma gangrenoso ${ }^{10}$.

A associação do pioderma gangrenoso, acne e artrite piogénica enquadra-se no síndrome PAPA ${ }^{16}$, uma doença autoinflamatória causada por mutações no gene PSTPIP1 localizado no cromossoma 15q24-q25.1 codificando a proteína 1 de ligação ao CD2 ${ }^{17}$. É uma doença de transmissão autossómica dominante, estando descritos 41 casos em 4 continentes $^{18}$.

Foi recentemente descrito uma síndrome autoinflamatória resultante da presença de pioderma gangrenoso, acne e hidrosadenite supurativa, PASH, estando ausente o componente de artrite do síndrome PAPA, mas presente a hidrosadenite supurativa. A nossa doente, no entanto, não tinha história de acne grave. Nesta síndrome não se detectou a mutação no gene PSTPIP1, nem as mutações MEFV, NLRP3 e TNFRSF1A, habitualmente associadas à maioria dos casos de febre mediterrânica familiar, síndrome periódico associado às criopirinas e os síndromes periódicos associados ao receptor 1 do factor de necrose tumoral. A única alteração detectada foi um aumento de repetição de microssatélites CCTG na área do promotor do PSTPIP1, também observados na síndrome de abcessos abdominais assépticos, que pode também surgir em associação com doença inflamatória intestinal, abcessos cutâneos e outras dermatoses neutrofílicas ${ }^{18}$.

$\bigcirc$ PG pode surgir em qualquer fase do desenvolvimento da HS, sendo uma associação de difícil resolução terapêutica com necessidade frequente de utilização de múltiplos fármacos para o seu tratamento $^{10}$.

Devido à raridade desta associação, as terapêuticas propostas baseiam-se em relatos e pequenas séries, estando descritos vários tratamentos possíveis. A corticoterapia oral mantém-se como primeira linha para o tratamento do PG, embora não seja frequentemente utilizada na HS devido ao curso crónico da mesma ${ }^{7}$. A corticoterapia fez parte dos esquemas de tratamento descritos, isoladamente ou em associação com imunossupressores, como a azatioprina e a ciclosporina, ou com antibióticos como a minociclina, rifampicina e clindamicina nas maiores séries descritas ${ }^{1,10}$.

A utilização de terapêuticas biológicas anti-TNF, com relatos de eficácia variável ${ }^{1,19}$, podendo ser combinadas com corticoterapia intralesional ${ }^{1}$, assim como a utilização de ciclosporina ${ }^{20}$, parecem ser promissores pela sua actuação em ambas as dermatoses. Os agentes antineutrofílicos clássicos, como a colchicina ${ }^{21}$ e a dapsona', têm sido ineficazes no tratamento desta entidade.

O antagonista do receptor da IL-1 - anakinra - parece ser eficaz no tratamento do síndrome PAPA ${ }^{22}$, com indução de resposta, ainda que parcial na síndrome $\mathrm{PASH}^{18}$ e ainda num caso desta associação', HS e PG, perspectivando-se como uma possível terapêutica.

Pretende-se com este caso descrever uma associação rara mas estabelecida, cuja etiologia não está ainda esclarecida mas com provável enquadramento numa síndrome autoinflamatória. O conhecimento da epidemiologia, com o aumento do número de casos relatados e os constantes desenvolvimentos no campo da investigação genómica possibilitarão, um melhor conhecimento desta patologia, com melhor intervenção e terapêutica alvo.

\section{BIBLIOGRAFIA}

1. Hsiao JL, Antaya RJ, Berger T, Maurer T, Shinkai K, Leslie KS. Hidradenitis suppurativa and concomitant pyoderma gangrenosum: a case series and literature review. Arch Dermatol. 2010;146(1 1):1265-70.

2. Brocq L. Nouvelle contribution a l'étude du phagédénisme géométrique. Ann Dermatol Syphiligr. 1916; 6: 1-39.

3. Brunsting LA, Goekerman WH, O'Leary PA. Pyoderma (ecthyma) gangrenosum: clinical and experimental observations in five cases in adults. Arch 


\section{Caso Clínico}

Dermatol. 1930; 22: 655-80.

4. Powell FC, Schroeter AL, Perry HO. Pyoderma gangrenosum. A review of 86 patients. Q J Med. 1985; 217: 173-86.

5. Adachi $Y$, Kindzelskii AL, Cookingham $G$, et al. Aberrant neutrophil trafficking and metabolic oscillations in severe pyoderma gangrenosum. J Invest Dermatol. 1998; 111: 259-68.

6. Brooklyn TN, Williams AM, Dunnill MG, Probert CS. T-cell receptor repertoire in pyoderma gangrenosum: evidence for clonal expansions and trafficking. Br J Dermatol. 2007;157(5):960-6.

7. Kurzen H, Kurokawa I, Jemec GB, Emtestam L, SeIlheyer K, Giamarellos-Bourboulis EJ, et al. What causes hidradenitis suppurativa? Exp Dermatol. 2008; 17(5):455-6; discussion 457-72.

8. Dvorak VC, Root RK, Macgregor RR. Host-defence mechanism in hidradenitis suppurativa. Arch Dermatol. 1977; 113: 450-3.

9. Fitzsimmons JS, Fitzsimmons EM, Bilbert G. Familial hidradenitis suppurativa: Evidence in favour of single gene transmission. J Med Genet. 1984; $21: 281$.

10. Ah-Weng A, Langtry JA, Velangi S, Evans CD, Douglas WS. Pyoderma gangrenosum associated with hidradenitis suppurativa. Clin Exp Dermatol. 2005; 30(6):669-71.

11. Mendonça CO, Griffiths CE. Clindamycin and rifampicin combination therapy for hidradenitis suppurativa. Br J Dermatol. 2006 ;154(5):977-8.

12. Jemec GB, Heidenheim M, Nielsen NH. The prevalence of hidradenitis suppurativa and its potential precursor lesions. J Am Acad Dermatol. 1996; 35(2 Pt 1):191-4.

13. Jemec GB. Hidradenitis suppurativa. J Cutan Med Surg. 2003;7(1):47-56.

14. Jemec GB. Body weight in hidradenitis suppurativa. In: Goldsmith L, Katz S, Gilchrest B, Paller A, Leffell
D, Wolff K, editors. Fitzpatrick's Dermatology in General Medicine. $8^{\text {th }}$ ed. New York: McGraw-Hill Professional; 2012. p.954.

15. Kromann C, Ibler KS, Kristiansen V, Jemec GB. The Influence of Body Weight on the Prevalence and Severity of Hidradenitis Suppurativa. Acta Derm Venereol. 2014.

16. Lindor NM, Arsenault TM, Solomon H, Seidman $C D$, McEvoy MT. A new autosomal dominant disorder of pyogenic sterile arthritis, pyoderma gangrenosum, and acne: PAPA syndrome. Mayo Clin Proc. 1997;72:611-5.

17. Yeon HB, Lindor NM, Seidman JG, Seidman CE. Pyogenic arthritis, pyoderma gangrenosum, and acne syndrome maps to chromosome 15q. Am J Hum Genet. 2000;66:1443-8.

18. Braun-Falco M, Kovnerystyy O, Lohse P, Ruzicka T. Pyoderma gangrenosum, acne, and suppurative hidradenitis (PASH)-a new autoinflammatory syndrome distinct from PAPA syndrome. J Am Acad Dermatol. 2012;66(3):409-15.

19. Reddick CL, Singh MN, Chalmers RJ. Successful treatment of superficial pyoderma gangrenosum associated with hidradenitis suppurativa with adalimumab. Dermatol Online J. 2010; 15;16(8):15.

20. Buckley DA, Rogers S. Cyclosporin-responsive hidradenitis suppurativa. J R Soc Med. 1995; 88: 289-90.

21. Raynor A, Askari AD. Behcet's disease and treatment with colchicine. J Am Acad Dermatol. 1980; 2: 396-400.

22. Brenner M, Ruzicka T, Plewig G, Thomas P, Herzer $P$. Targeted treatment of pyoderma gangrenosum in PAPA (pyogenic arthritis, pyoderma gangrenosum and acne) syndrome with the recombinant human interleukin-1 receptor antagonist anakinra. $\mathrm{Br} \mathrm{J}$ Dermatol. 2009;161:1199-201. 